\title{
An Integrated CEA Approach for Color Light Source Estimation
}

\author{
Harpreet Kaur \\ Department of Computer Engineering \& Technology, Guru Nanak Dev University, Amritsar, 143001, India \\ E-mail: harpreetrajput44@gmail.com
}

Sandeep Sharma

Professor, Department of Computer Engineering \& Technology, Guru Nanak Dev University, Amritsar, 143001, India

E-mail: sandeep.cse@gndu.ac.in

\begin{abstract}
Color constancy is an element of human vision framework which guarantees that the apparent color of items under fluctuating light conditions generally remains constant. It is fundamentally used to eliminate the color cast in the picture. Color Cat is a quick and precise learning-based methodology for accomplishing computational color constancy. However, despite everything it confronts a few limitations like poor brightness due to normalization used. Furthermore it doesn't promise edge preservation. So to overcome these issues a CEA strategy has been proposed which is a hybrid model based on Color Cat, Edge preservation filter and Adaptive histogram Equalization. As Adaptive histogram Equalization is exceptionally valuable for contrast improvement and edges are protected by edge preservation filter. Experimental results show that the proposed CEA approach outperforms over existing techniques.
\end{abstract}

Index Terms - Color constancy, illuminant estimation, performance evaluation, Adaptive histogram Equalization, Edge preservation filter, CLAHE(Contrast Limited Adaptive Histogram Equalization)

\section{INTRODUCTION}

In Image Processing Color Constancy is the capability to see color of articles regardless of the shade of the light source [1]. Fortunately, individuals have the limit of color steadiness, they see the same shade of a thing independent of extensive contrasts in enlightenment. A similar shading unfaltering quality limit is imperative for various PC vision assignments, for instance, object affirmation, video recuperation and scene classification. The shade of a light source sways object tints in the scene. These changed color qualities may show undesirable effects in electronic pictures. Other than it may conversely impact the execution of PC vision systems for different applications [2].The purpose of shading consistent quality is to conform the consequence of the illuminant shading, either by enlisting invariant components or by changing the information picture such that the consequences of the shade of the light source are cleared [3]. Color Constancy techniques are isolated into three ways: low-level statistics based strategies, gamut based mapping and learning based strategies. The first kinds of calculations are strategies that are connected to any picture without the requirement for training. Before the illuminant can be evaluated, a model should be prepared for second and third sort of calculations. [4].Low level measurements based techniques incorporate White Patch Retinex: It is one among the essential shading consistency approaches considering single light source and ventures the things that are truly white i.e. the lightest patch within the scene. It expect that sudden change in reflectance model causes the conformity in chromaticity [4]. Gray World depends on assumption that the ordinary reflectance of surfaces is color less and by applying Color balancing algorithms picture it is expected to have uniform scene brightening [5]. In Gray Edge Hypothesis, As contrasted with 4associated, there is accessibility of more points of interest for picture rectification in 8 -associated. It is not considered as productive on the grounds that each pixel is considering its 4-neighboring pixels just for the assessment [6]. Color Rabbit is extremely successful Retinex-based strategy for brightening estimation. Both precision and calculation expense can just be controlled by a few parameters and the technique's exactness is appeared to be powerful to change of parameter qualities [7].Learning based calculations incorporate Gamut mapping calculation, that is a basic, quick calculation for figuring the arrangement of conceivable lights steady with a given picture. The yield of array mapping is an arrangement of 2-D practical chromaticity maps[8].Other learning-based algorithms include color-by correlation is variant of gamut mapping where correlation matrix replaces the canonical gamut.Calculation of correlation matrix is performed for considered illuminations and the illumination with the highest probability is selected [9].This approach needs large amount of training data and can provide good results when trained correctly. Bayesian formulation based method models the variability of reflectance and light source as irregular variables and illumination estimation from the posterior distribution on the image intensity have also been proposed. It has been demonstrated the need for precise priors for reflectance and illumination [10].In Exemplar- 
based learning approach, test image figures nearest neighbor models for each surface and enlightenment estimation is done taking into account looking at the statistics of pixels [11]. Color constancy using saturation weighting use distinctive approaches in accordance to saturation values from many observations and ultimately incorporated a saturation weighting function into the already present techniques [12].By using Sparse Coding for Enlightenment Estimation, the photo's scene content information is joined with its shading dissemination to perform perfect light estimation [13]. 3D geometry models are utilized to review which color consistency technique is suitable to use for the particular geometrical districts in pictures [14].Object shading models from multi-view confinements can misuse picture correspondences acquired by different arrangement systems, and it has indicated cases taking into account coordinating close-by area highlights [15]. Color Cat is speedy and exact learning-based procedure to achieve computational shading consistency. Color Cat is quick and precise learning-based technique to accomplish computational color consistency. Relationship between regularity in the desirable illumination colors and modified color histograms is used in color cat. The principle step in accomplishing computational color consistency is the illumination estimation of color light source that is further used to eliminate the color cast effect. It performs illumination estimation by using information provided by image pixels and adjust the distinctive histograms. It compares the illumination estimation with the truth ground values [16].

This paper is structured as follows: Section2 presents the related work in the field of color constancy methods. Section 3 defines the proposed work which includes the steps of proposed methodology and Section 4 includes the parameters on the basis of which experimental results are drawn. Section 5 includes the experimental set up consisting the results in the form of tables and bar graphs of existing and proposed method and finally conclusions are provided in section 6 .

\section{RELATED WORK}

Choudhury, et al.(2010) [21] have explained Color Constancy systems as disengaged in three ways: lowlevel statistics based methodologies, gamut based mapping and learning based techniques. Authors have proposed another strategy to accomplish color constancy taking into account the measurements of pictures with color cast. Pictures with color cast have standard deviation of one color channel essentially not quite the same as that of other color channel and it is likewise appropriate to nearby fixes of pictures and proportion of the greatest and least standard deviation of color channels.

Gijsenij, et al. (2011)[2] have demonstrated Color Constancy methodology by utilizing Natural picture statistics and scene semantics. Natural picture statistics are utilized to analyze the best significant properties of color pictures. On the basis of these properties, the color constancy methodology is selected for a particular picture.
Weibull parameterization is used for acquiring picture properties. Experiment has shown that on a data set involving more than 11,000 images, There can be an increment of $20 \%$ in color constancy performance in terms of median angular error.

Gijsenij et al.(2012)[1] have amplified the current calculations by applying color constancy locally to picture patches, instead of the whole picture. After the estimation of neighborhood illuminant, these estimates are joined into more vigorous estimations, Experiments has shown that the proposed method results in reduction of the influence of two light sources concurrently present in one picture. In the event that the chromatic distinction between two illuminants is more than $1^{\circ}$, It outperforms from the algorithms based on the uniform light-source assumption .Otherwise, when the chromatic difference is less than $1^{\circ}$ and the scene considered to contain single uniform light source, the performance of the proposed methodology is similar to global color constancy techniques.

Chakrabarti, et al.(2012)[20] have presented Color Constancy with Spatio-Spectral Statistics. They acquainted the model to eliminate the color cast produced by the spectral distribution of the dominating scene illuminant from a picture. They built up a model for spatial distribution of colors in white balanced images and after that by utilizing this model ,illumination parameters are figured. Exploratory evaluation on standard information sets proposes that the proposed methodology outperforms than traditional methods.

Gijsenij, et al.(2012)[19] have enhanced Color Constancy by Photometric Edge Weighting. This methodology make utilization of picture derivatives for illumination estimation. They have demonstrated an execution assessment of edge-based color constancy utilizing distinctive edge types. This execution assessment determined that specular and shadow edge sorts are more important than material edges for the illuminant estimation. Thus, the iterative weighted GrayEdge calculation is schemed . This iterative weighted Gray-Edge calculation lowers the median angular error with around 25 percent and when contrasted with edge based Color Constancy it has indicated upgrades in angular error up to 11 percent.

Vazquez, et al.(2012)[9] have shown Color Constancy by Category Correlation. They have proposed perceptual limitations that are processed on the corrected pictures. They have characterized the category hypothesis that weights the arrangement of practical illuminants to outline the corrected picture onto particular colors and also proposed a quick implementation that permits the testing of a large arrangement of illuminants. Test results have demonstrated that this strategy rivals current stateof-art execution without the requirement for training algorithmic framework. Furthermore, the strategy can be utilized as a system to embed top-down data from different sources.

Vaezi , et al.(2012) [22] have characterized the White Patch Gamut as another augmentation to the Gamut Mapping Color Constancy strategy, containing the 
brilliant pixels of the picture and this strategy beats array mapping techniques.

Rezagholizadeh et al. (2013) [18] have proposed EdgeBased and powerful Chromaticity Spatio-spectral Models for Color Constancy. They explained that quick and exact estimation of the conversion forced by the illuminant to the color of a picture taken under that illuminant is of great significance in real-time computational color constancy operations. They exhibited evaluation of the performance of the proposed technique on a synthetic color picture database.

Marin-Franch et al. (2013)[17] have evaluated data from color of picture with two goals. Firstly ,to determine estimators of the accessible data and the data recovered under various illuminations from the color values at every point in pictures of a scene. Secondly, applying these estimators to simulations of pictures acquired with five arrangements of sensors utilized in computerized cameras and with the cone photoreceptors of the human eye.

Banic et al.(2014)[7] have clarified low-level statistics based color constancy calculation for illumination estimation and tried. They consolidated numerous local illumination estimations by utilizing a new methodology into a global one. The proposed calculation is tried on a few accessible databases and it performs very well than all other color constancy strategies as far as execution pace and exactness are concerned.

Joze, etal.(2014) [11] have analyzed Color Constancy and Multiple Illumination as the model based learning. They dissected that as opposed to focusing on surfaces in the picture and addressing the color constancy issue by unsupervised learning for every training surface in training pictures. They proposed closest neighbor models for every surface in a test picture and estimate its illumination on the basis of comparing the measurements of pixels related to closest neighbor surfaces and the target surface. They have demonstrated a strategy to beat the various illuminant situation and test is conducted utilizing a numerous illuminant color constancy on pictures with two different sources of illumination.

Banic et al. (2015)[16] have proposed the Color Cat (CC), a very fast and best learning-based technique to accomplish computational color constancy. This method utilizes the relationship between transformed color histograms and the regularity in the possible illumination colors. The proposed method outperformed from the other color constancy methods in a way accuracy and computation cost is concerned.

Banic, et al. (2015)[23] have exhibited how a current technique can be simplified by utilizing effective elements for color constancy taking into account the red chromaticity.

Sahani, et al. (2015) [24] have planned an installed framework with changed contrast limited adaptive histogram equalization technique for real-time image enhancement and this framework has shown remarkable results than existing methodologies.

Muniyappan, et al. (2013) [26] Authors have characterized new methodology for picture enhancement by utilizing CLAHE technique that results in a better contrast pictures.

Khan, et al.(2013)[27]Creators have executed Balanced CLAHE for Adaptive Dynamic Range Compression (ADRC) of real time medical pictures and this proposed methodology has demonstrated better outcomes as far as latency and perceptibility parameters are concerned.

Iwanami, et al.(2012) [28] Authors have used the DRSHE technique to the part of the picture so as to enhance the regional picture contrast in short computational time.

Lidong, et al.(2015) [29] have displayed a novel picture upgrade strategy, named CLAHE-discrete wavelet change (DWT), which joins the CLAHE with DWT.

Kaur, et al.(2015)[31] have proposed Biorthogonal Wavelet Transform by Utilizing Bilateral Filter and Adaptive Histogram Equalization and by the use of this methodology, experimental results has shown improvement in fusion quality by the reduction in loss of important data available in separate pictures

Hameed, et al.(2011) [30] have characterized the strategy that includes two stages. The initial step is to perform AHE to enhance the signal contrast in a discriminative way and then the histogram of the input picture is taken into consideration. After that recognition of irregularity of image intensity is performed and on recognition it is eliminated by utilizing a CLAHE technique.

Begill et al. (2016) [25] have planned an installed framework with changed contrast limited adaptive histogram equalization technique for real-time image enhancement and this framework has shown remarkable results than existing methodologies.

\section{PROPOSED WORK}

A New CEA algorithm has been proposed which provides the complete information about Color light Source estimation by using Color Cat and edge preservation filter which presents superior results than existing Color Cat technique. The implementation process of proposed algorithm has been demonstrated in Fig. 1 and Steps of algorithm are clarified below:

Step1: Take the image and construction of the histogram of the input image is done.

Step2: After obtaining the histogram of selected image, mean values of red, green and blue channel are evaluated.

Step 3: Evaluate the global mean value from the mean values of red, green and blue channel obtained in previous step.

Step 4: Restore red, green and blue using global mean value with the help of Color Cat algorithm.

Step 5: Application of Edge preservation filter on the selected image is performed.

Step 6: After the application of edge preservation filter Contrast limited adaptive histogram equalization (CLAHE) is performed on the input image. 
Step 7: Finally Evaluation of parameters is performed and restored image is returned.

Step 8: Final image is acquired with improved quality and preserved edges as the output of this proposed methodology.

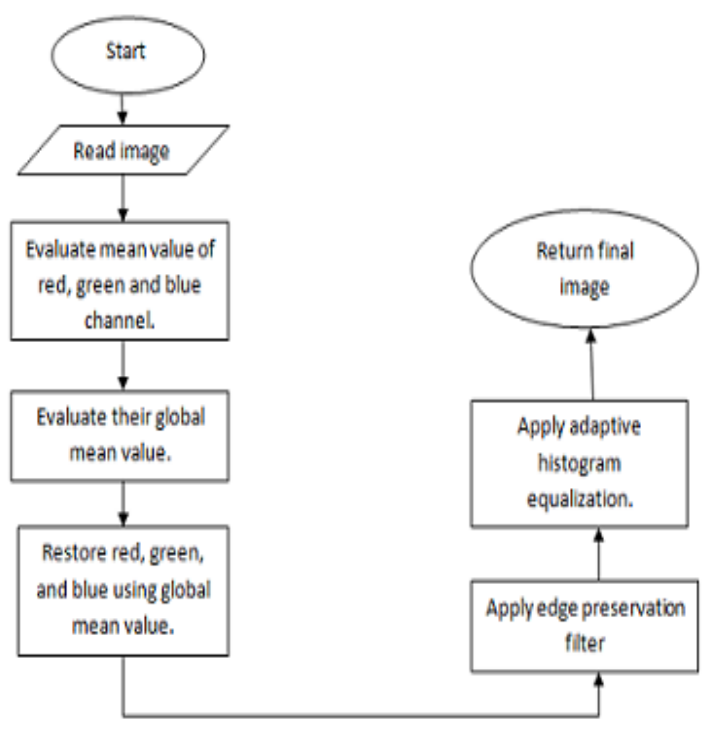

Fig.1. Flowchart of proposed algorithm

\section{Performance Metrices}

The investigation of picture quality is finished by subjective assessment alongside target assessment. For subjective assessment, it is elusive distinction in the middle of proposed and existing technique. Along these lines, execution measurements have been utilized to dissect the execution of color pictures. Performance metrices are MSE, RMSE, PSNR and BER. It has demonstrated that the execution of the arranged calculation is superior than the existing techniques.

\section{A. Mean Square Error (MSE)}

Mean square error is utilized to assess picture quality list. High estimation of mean square shows that nature of picture is poor.

$$
\operatorname{MSE}=\frac{1}{I R} \sum_{i=1}^{I} \sum_{r=1}^{R}[P(i, r)-Q(i, r)]^{2}
$$

Where $\mathrm{P}(\mathrm{i}, \mathrm{r})$ is value of pixel of input image.

$\mathrm{Q}(\mathrm{i}, \mathrm{r})$ is value of pixel of reconstructed image.

\section{B. Root Mean Square Error (RMS)}

Substantial estimation of root mean square error demonstrates that nature of picture is unfavorable. As RMSE must be maximized, therefore the key goal would be to decrease the RMSE around possible. It is computed as:

$$
\mathrm{RMSE}=\sqrt{\frac{1}{I R} \sum_{i=1}^{I} \sum_{r=1}^{R}[P(i, r)-Q(i, r)]^{2}}
$$

\section{Peak Signal to Noise Ratio (PSNR)}

PSNR is basically the ratio that is used as a performance metric between input image and reconstructed image. It is computed as:

$$
\text { PSNR }=10 \log _{10}\left(\frac{255}{M S E}\right)^{2}
$$

\section{Bit Error Rate (BER)}

Bit error rate is used in accessing systems that transfer digital data from one place to another and is defined as the number of bit errors divided by the total number of transferred bits during a specific time interval.

\section{EXPERIMENTAL SETUP}

The proposed CEA algorithm has been tested on ten color images and the results are evaluated. Different parameters like MSE, RMSE, PSNR, BER are used for evaluating the performance of these methods. Ten experimental images are considered and their improved values of parameters are shown below and Results of applying proposed CEA method over the existing Color Cat technique has clearly shown in the form of images in Fig.6(a) and Fig.6(b).These includes ten input images along with their results. Fig. 6(a) and Fig. 6(b) are placed at the end of REFERENCES section.

Table 1. Mean Square Error

\begin{tabular}{|c|c|c|}
\hline Input images & $\begin{array}{c}\text { Existing Color Cat } \\
\text { Algorithm }\end{array}$ & $\begin{array}{c}\text { Proposed } \\
\text { CEA Algorithm }\end{array}$ \\
\hline 1 & 463.7641 & 9.8412 \\
\hline 2 & 777.5763 & 44.6547 \\
\hline 3 & 525.0034 & 55.7444 \\
\hline 4 & 377.1125 & 116.0597 \\
\hline 5 & 224.4842 & 125.4587 \\
\hline 6 & 108.5944 & 94.9016 \\
\hline 7 & 20.3155 & 15.1895 \\
\hline 8 & 340.0370 & 47.2816 \\
\hline 9 & 159.8068 & 139.1771 \\
\hline 10 & 639.2008 & 28.0047 \\
\hline
\end{tabular}

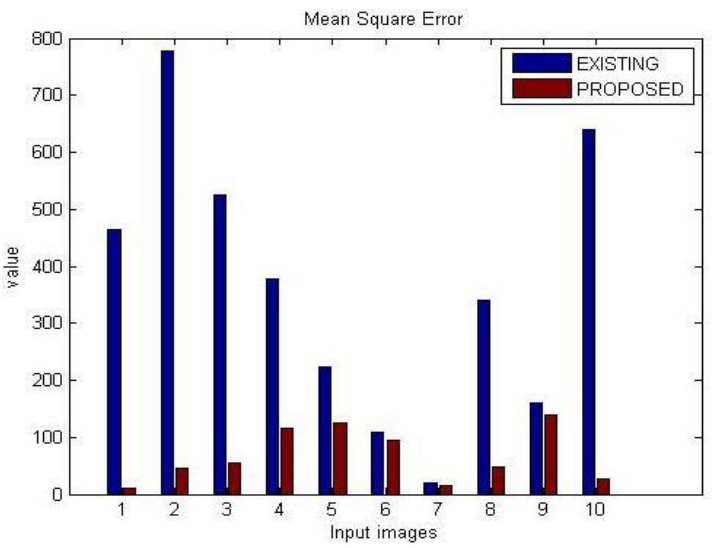

Fig.2. Mean Square Error 
As mean square error has been decreased which means proposed CEA algorithm is featuring the better benefits compared to the existing color cat technique as mean square error is less in all the samples (as shown in Table 1)

Fig. 2 is representing a bar graph of mean square values of ten images that are considered as a sample. Blue colored bar shows the MSE values of existing method while red colored bar shows values of proposed CEA technique. It is clearly shown that mean square error is less in all the samples by using proposed technique. Hence, the results are better than existing method.

Table 2. Root mean Square Error

\begin{tabular}{|c|c|c|}
\hline Input images & $\begin{array}{c}\text { Existing Color CAT } \\
\text { Algorithm }\end{array}$ & $\begin{array}{c}\text { Proposed CEA } \\
\text { Algorithm }\end{array}$ \\
\hline 1 & 21.5352 & 3.1371 \\
\hline 2 & 27.8492 & 6.6824 \\
\hline 3 & 22.9130 & 7.4662 \\
\hline 4 & 19.4194 & 10.7731 \\
\hline 5 & 14.9828 & 11.2008 \\
\hline 6 & 10.4209 & 9.7422 \\
\hline 7 & 4.5073 & 3.8974 \\
\hline 8 & 18.4401 & 6.8762 \\
\hline 9 & 12.6415 & 11.7973 \\
\hline 10 & 25.2824 & 5.2919 \\
\hline
\end{tabular}

Table 2 has clearly shown that the RMSE is minimal in case of the proposed method. Thus proposed CEA algorithm is providing better effects compared to the existing technique. Root Mean squared error between the reference image and the final image is as shown in Fig. 3.

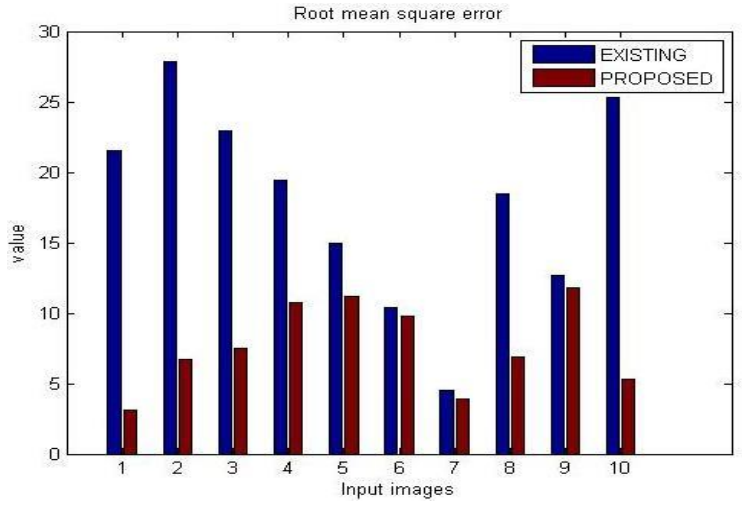

Fig.3. Root Mean Square Error

Fig. 3 is representing a bar graph of root mean square values of ten images that are considered as a sample. Blue colored bar shows the RMSE values of existing method while red colored bar shows values of proposed CEA technique. It is clearly shown that the root mean square error is less in all the samples by using proposed technique. Hence, results are better than existing method.
Table 3. Peak Signal Noise Ratio

\begin{tabular}{|c|c|c|}
\hline Input images & $\begin{array}{c}\text { Existing Color CAT } \\
\text { Algorithm }\end{array}$ & $\begin{array}{c}\text { Proposed CEA } \\
\text { Algorithm }\end{array}$ \\
\hline 1 & 21.4678 & 38.2003 \\
\hline 2 & 19.2346 & 31.6321 \\
\hline 3 & 20.9292 & 30.6688 \\
\hline 4 & 22.3661 & 27.4840 \\
\hline 5 & 24.6189 & 27.1458 \\
\hline 6 & 27.7727 & 28.3577 \\
\hline 7 & 35.0525 & 36.3154 \\
\hline 8 & 22.8154 & 31.3839 \\
\hline 9 & 26.0949 & 26.6951 \\
\hline 10 & 20.0744 & 33.6585 \\
\hline
\end{tabular}

Higher value of PSNR leads to high quality of reconstructed image and Table 3 shows that value of PSNR is high in proposed technique and as shown in Fig. 4.

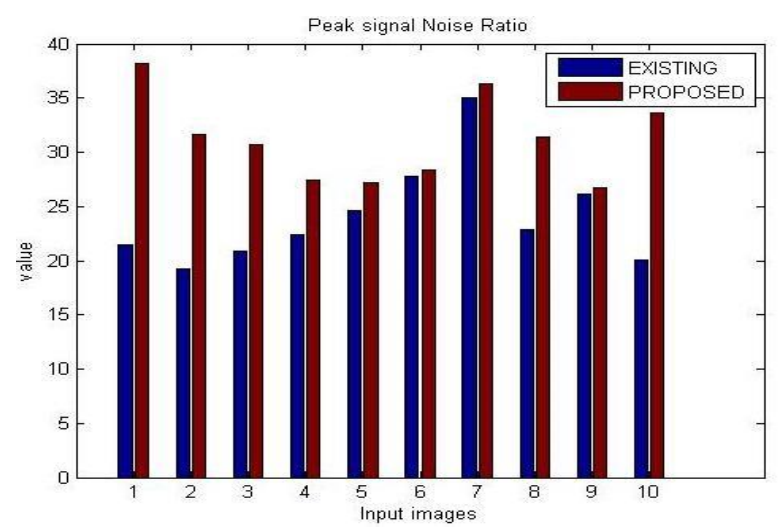

Fig.4. Peak Signal Noise Ratio

Table 4. Bit Error Rate

\begin{tabular}{|c|c|c|}
\hline Input images & $\begin{array}{c}\text { Existing Color CAT } \\
\text { Algorithm }\end{array}$ & $\begin{array}{c}\text { Proposed CEA } \\
\text { Algorithm }\end{array}$ \\
\hline 1 & 0.0466 & 0.0262 \\
\hline 2 & 0.0520 & 0.0316 \\
\hline 3 & 0.0478 & 0.0326 \\
\hline 4 & 0.0447 & 0.0364 \\
\hline 5 & 0.0406 & 0.0368 \\
\hline 6 & 0.0360 & 0.0353 \\
\hline 7 & 0.0285 & 0.0275 \\
\hline 8 & 0.0438 & 0.0319 \\
\hline 9 & 0.0383 & 0.0375 \\
\hline 10 & 0.0498 & 0.0297 \\
\hline
\end{tabular}

Fig. 4 is representing a bar graph of peak signal to noise ratio of ten images that are considered as a sample. Blue colored bar shows the PSNR values of existing method while red colored bar shows values of proposed CEA technique. It is clearly shown that PSNR is more in all the samples by using proposed technique. 
As shown in Table 4, the value for BER is lower by using proposed technique than the existing method. Thus proposed methodology is showing better performance.

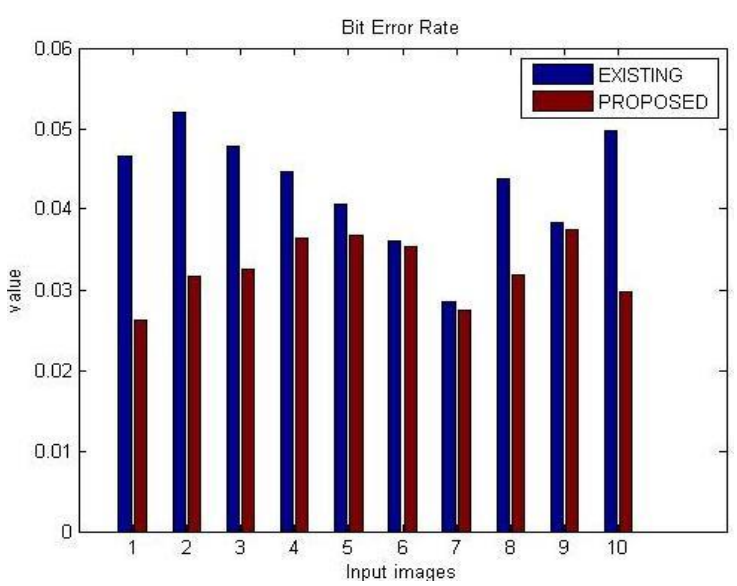

Fig.5. Bit Error Rate

Fig. 5 is representing a bar graph of bit error rate values of ten images that are considered as a sample. Blue colored bar shows the BER values of existing method while red colored bar shows values of proposed CEA technique. As it has been clearly shown that as BER is less in all the samples by using proposed technique. Hence, results shows greater improvement than existing technique.

\section{CONCLUSION AND FUTURE WORK}

The proposed CEA strategy is an integrated approach which is based on Color Cat, Adaptive histogram equalization and Edge preservation filter so as to overcome the restrictions of existing technique. Experimental results shows that parameter BER value has been decreased to 0.01026 , parameter MSE value decreased to 295.9609, RMSE value decreased to 10.11272 and PSNR value increased to 7.11151 by using proposed technique. Hence the proposed CEA algorithm is better than existing technique in terms of evaluated parameters. This work has not been considered for the use of fuzzy based enhancement. So in near future, one can utilize fuzzy membership functions to enhance the quality of picture.

\section{REFERENCES}

[1] A.Gijsenij, Rui Lu,T.Gevers, "Color Constancy for Multiple Light Sources," in Image Processing, IEEE Transactions on , vol.13, no.2, pp.697-707, Feb. 2012

[2] A.Gijsenij, T.Gevers, "Color Constancy Using Natural Image Statistics and Scene Semantics," in Pattern Analysis and Machine Intelligence, IEEE Transactions on , vol.33, no.4, pp.687-698, April 2011

[3] J. van de Weijer, T. Gevers and A. Gijsenij, "Edge-Based Color Constancy," in IEEE Transactions on Image Processing, vol. 16, no. 9, pp. 2207-2214, Sept. 2007

[4] N.Banic, S.Loncaric, "Improving the white patch method by subsampling," in Image Processing (ICIP), 2014 IEEE International Conference on , vol., no., pp.605-609, 27-30
Oct. 2014

[5] "Critical Analysis of Different Color Constancy Algorithm", [ONLINE AVAILABLE]: www.ijetae.com

[6] A.Gijsenij,T.Gevers, J.Van de Weijer, "Computational Color Constancy: Survey and Experiments," in Image Processing, IEEE Transactions on , vol.20, no.9, pp.24752489, Sept. 2011

[7] N.Banic, S.Loncaric, "Color Rabbit: Guiding the distance of local maximums in illumination estimation," in Digital Signal Processing (DSP), 2014 19th International Conference on , vol., no., pp.345-350, 20-23 Aug. 2014

[8] G. Finlayson and S. Hordley, "Improving gamut mapping color constancy," in IEEE Transactions on Image Processing, vol. 9, no. 10, pp. 1774-1783, Oct 2000

[9] J.Vazquez-Corral, M.Vanrell, R.Baldrich, F.Tous,"Color Constancy by Category Correlation," in Image Processing, IEEE Transactions on , vol.13, no.4, pp.1997-2007, April 2012

[10] M.P.Lucassen,T.Gevers,A.Gijsenij, "Color fidelity of chromatic distributions by triad illuminant comparison," in IVMSP Workshop, 2011 IEEE 10th ,vol., no.,pp.1-6, 16-17 June 2011

[11] H.R.V.Joze, M.S.Drew, "Exemplar-Based Color Constancy and Multiple Illumination," in Pattern Analysis and Machine Intelligence, IEEE Transactions on , vol.36, no.5, pp.860-873, May 2014

[12] Hyunchan Ahn, Soobin Lee, Hwang Soo Lee, "Improving color constancy by saturation weighting," in Acoustics, Speech and Signal Processing (ICASSP), 2013

[13] Bing Li, Weihua Xiong, Weiming Hu, Houwen Peng, "Illumination Estimation Based on Bilayer Sparse Coding," in Computer Vision and Pattern Recognition (CVPR), 2013 IEEE Conference on , vol., no., pp.14231429, 23-28 June 2013

[14] N.Elfiky, T.Gevers, A. Gijsenij, J.Gonzalez, "Color Constancy Using 3D Scene Geometry Derived From a Single Image," in Image Processing, IEEE Transactions on , vol.23, no.9, pp.3855-3868, Sept. 2014

[15] T.Owens,K.Saenko, A. Chakrabarti, Ying Xiong,T. Zickler,T. Darrell, "Learning object color models from multi-view constraints," in Computer Vision and Pattern Recognition (CVPR), 2011 IEEE Conference on , vol no., pp.169-176, 20-25 June 2011

[16] N.Banic, S.Loncaric, "Color Cat: Remembering Colors for Illumination Estimation," in Signal Processing Letters, IEEE , vol.22, no.6, pp.651-655, June 2015[16] Banic, N. Loncaric, S., "Color Cat: Remembering Colors for Illumination Estimation," in Signal Processing Letters, IEEE , vol.22, no.6, pp.651-655, June 2015

[17] Marín-Franch, I.; Foster, D.H., "Estimating Information from Image Colors: An Application to Digital Cameras and Natural Scenes," in Pattern Analysis and Machine Intelligence, IEEE Transactions on , vol.35, no.1, pp.7891, Jan. 2013

[18] Rezagholizadeh, M.; Clark, J.J., "Edge-Based and Efficient Chromaticity Spatio-spectral Models for Color Constancy," in Computer and Robot Vision (CRV), 2013 International Conference on , vol., no., pp.188-195, 28-31 May 2013

[19] Gijsenij, A.; Gevers, T.; van de Weijer, J., "Improving Color Constancy by Photometric Edge Weighting," in Pattern Analysis and Machine Intelligence, IEEE Transactions on, vol.34, no.5, pp.918-929, May 2012

[20] Chakrabarti, A.; Hirakawa, K.; Zickler, T., "Color Constancy with Spatio-Spectral Statistics," in Pattern Analysis and Machine Intelligence, IEEE Transactions on , vol.34, no.8, pp.1509-1519, Aug. 2012 
[21] A. Choudhury and G. Medioni, "Color Constancy Using Standard Deviation of Color Channels," Pattern Recognition (ICPR), 2010 20th International Conference on, Istanbul, 2010, pp. 1722-1726

[22] H. R. Vaezi ,Joze and M. S. Drew, "White Patch Gamut Mapping Colour Constancy," Image Processing (ICIP), 2012 19th IEEE International Conference on, Orlando, FL, 2012, pp. 801-804.

[23] N. Banić and S. Lončarić, "Using the red chromaticity for illumination estimation," Image and Signal Processing and Analysis (ISPA), 2015 9th International Symposium on, Zagreb, 2015, pp. 131-136

[24] M. Sahani, S. K. Rout, L. M. Satpathy and A. Patra, "Design of an embedded system with modified contrast limited adaptive histogram equalization technique for real-time image enhancement," Communications and Signal Processing (ICCSP), 2015 International Conference on, Melmaruvathur, 2015, pp.0332-0335

[25] A.Begill and S.Arora, "An Improved DCT Based Image Fusion Using Saturation Weighting And Joint Trilateral Filter,"Advances in intelligent computing 384,Springer International Publishing Switzerland 2016, pp 447-457

[26] S. Muniyappan, A. Allirani and S. Saraswathi, "A novel approach for image enhancement by using contrast limited adaptive histogram equalization method," Computing, Communications and Networking Technologies (ICCCNT),2013 Fourth International Conference on, Tiruchengode, 2013, pp. 1-6.

[27] R. Khan, M. Talha, A. S. Khattak and M. Qasim, "Realization of Balanced Contrast Limited Adaptive Histogram Equalization (B-CLAHE) for Adaptive Dynamic Range Compression of real time medical images," Applied Sciences and Technology (IBCAST), 2013 10th International Bhurban Conference on, Islamabad,2013,pp.117-121

[28] T. Iwanami, T. Goto, S. Hirano and M. Sakurai, "An adaptive contrast enhancement using regional dynamic histogram equalization," 2012 IEEE International Conference on Consumer Electronics (ICCE), LasVegas,NV,2012,pp.719-722.

[29] H. Lidong, Z. Wei, W. Jun and S. Zebin, "Combination of contrast limited adaptive histogram equalisation and discrete wavelet transform for image enhancement," in IET Image Processing, vol. 9, no.10,pp.908-915,10,2015

[30] Z. Hameed and C. Wang, "Edge detection using histogram equalization and multi-filtering process," 2011 IEEE International Symposium of Circuits and Systems (ISCAS), Rio de Janeiro, 2011, pp. 1077-1080.

[31] Savroop Kaur, Hartej S. Dadhwal,"Biorthogonal Wavelet Transform Using Bilateral Filter and Adaptive Histogram Equalization", IJISA, vol.7, no.3, pp.37-43, 2015. DOI: 10.5815/ijisa.2015.03.05
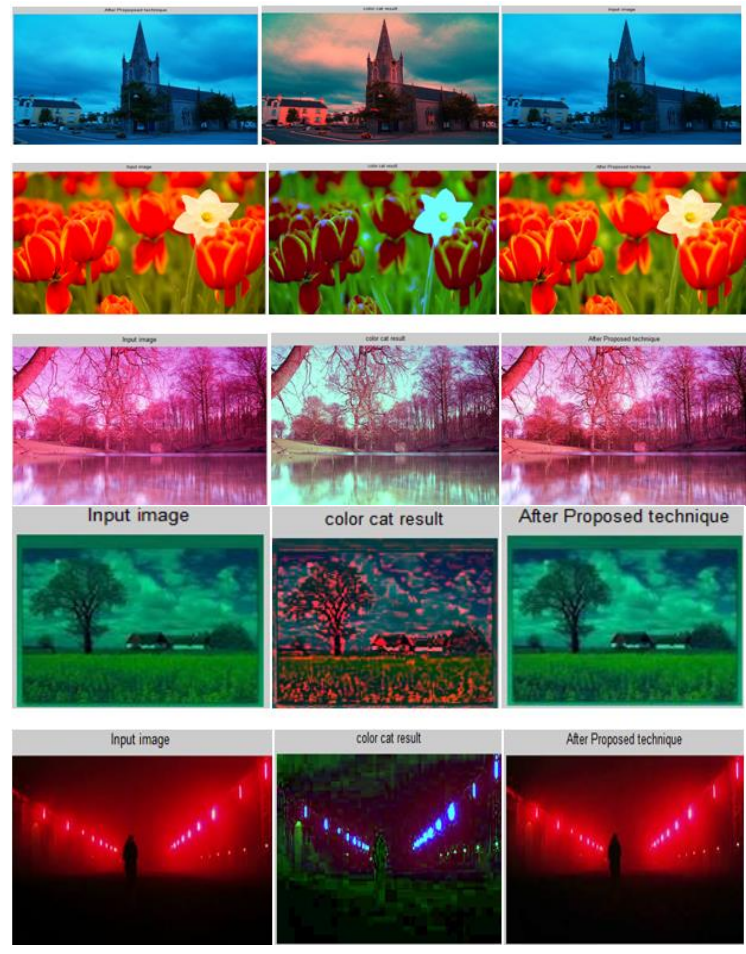

Fig.6.(a): Results obtained after applying proposed CEA technique and Color cat on the sample of images
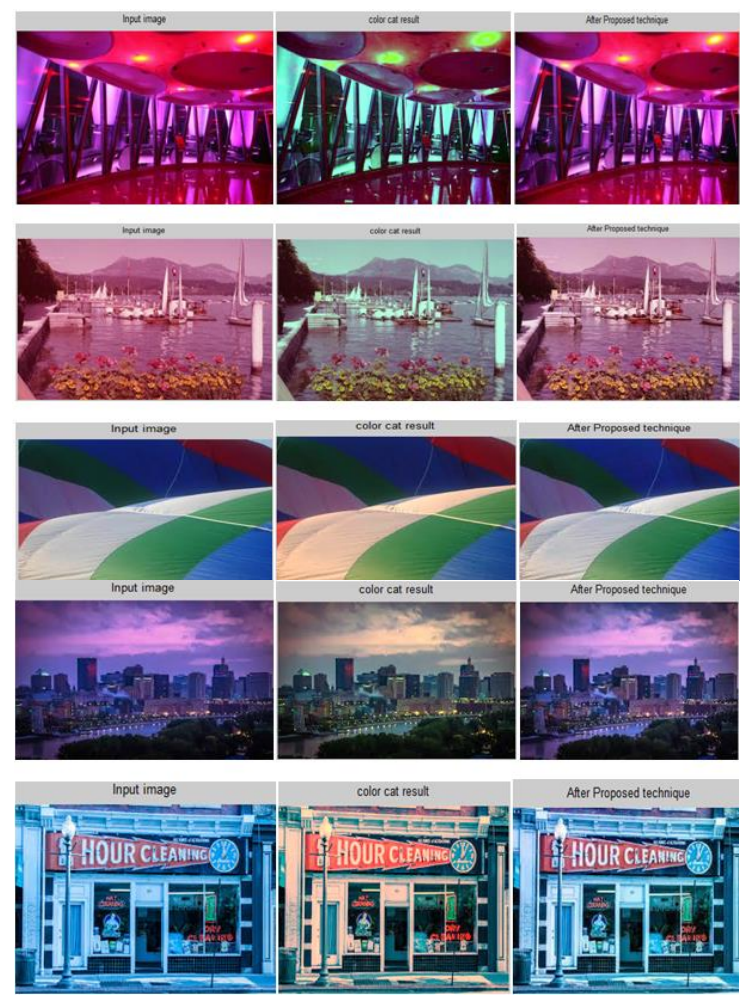

Fig.6.(b): Results obtained after applying proposed CEA technique and Color cat on the sample of ten images 


\section{Authors' Profiles}

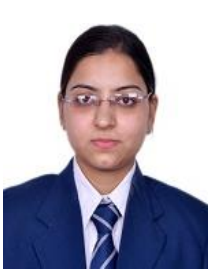

Harpreet Kaur (born April 04, 1992) is a Student, pursuing M.Tech in Software Systems at Department of Computer Engineering \& Technology, Guru Nanak Dev University Amritsar. Her main interest area is Digital Image Processing

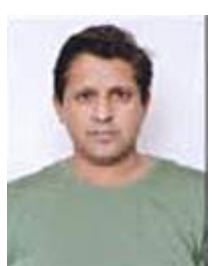

Sandeep Sharma graduated with B.E (Computer) degree from the University of Pune, received the M.E(Computer) from Thapar University Patiala and received his $\mathrm{PhD}$ degree from Guru Nanak Dev University Amritsar. He is Professor and Head of Department of Computer Engineering and Technology, Guru Nanak Dev University Amritsar. He is Chief Security Information Officer as well as Nodal Officer of Digital India Week. He is the Network as well as mail Administrator of the Guru Nanak Dev University .He has many research publications in the areas of parallel processing, wireless sensor networks and Cloud computing.

How to cite this paper: Harpreet Kaur, Sandeep Sharma,"An Integrated CEA Approach for Color Light Source Estimation", International Journal of Information Technology and Computer Science(IJITCS), Vol.9, No.2, pp.58-65, 2017. DOI: 10.5815/ijitcs.2017.02.07 